\title{
因HAD
}

ISSN-L: 2530-5115

DOI: http://doi.org/10.22585/hospdomic.v3i2.71

\section{Desarrollo de una Aplicación Móvil para la Evaluación Nutricional}

\section{Development of a Mobile Application for Nutritional Evaluation}

Carmina Wanden-Berghe', Luis Modesto Alvarez Sabucedo², Víctor M Alonso-Rorís ${ }^{3}$.

1. Hospital General Universitario de Alicante. Instituto de Investigación Sanitaria y Biomédica de Alicante ISABIAL-FISABIO.

2. Escola de Enxeñaría de Telecomunicación de la Universidad de Vigo, Vigo, España.

3. DataSpartan. Wood Street, London, United Kingdom.

Correspondencia/Correspondence

Carmina Wanden-Berghe Lozano

Hospital General Universitario de Alicante, Pintor

Baeza s/n, Alicante 03010

carminaw@telefonica.net

Recibido/Received

31.03 .2019

Aceptado/Accepted

31.03.2019
Conflicto de Intereses/Competing interest Los autores, en su totalidad declaran que no hay ningún posible conflicto de interés

Financiación/Funding

Se ha recibido apoyo financiero de la empresa Persan Farma SL para la realización de la aplicación móvil.

Agradecimientos/Acknowledgments

A los profesionales que han participado desinteresadamente con sus opiniones.

CÓMO CITAR ESTE TRABAJO | HOW TO CITE THIS PAPER

Wanden-Berghe C, Alvarez Sabucedo LM. Alonso-Rorís VM, Desarrollo de una Aplicación Móvil para la Evaluación Nutricional. Hosp Domic. 2019;3(2): 139-47. 


\section{RESUMEN}

Introducción: el uso de las llamadas apps o aplicaciones móviles está revolucionando el modo de trabajar en la práctica clínica. Aunque, actualmente es posible identificar gran cantidad de estas, en la revisión de la literatura no se hallan opciones como la propuesta por los autores.

Objetivo: desarrollar una herramienta de uso sencillo que permita una orientación diagnóstica sobre el estado nutricional, con soporte normativo, disponible en diferentes plataformas móviles y pensanda para la ayuda al profesional sanitario en la praxis clínica.

Métodos: Se realizó un consenso de expertos (grupo nominal y técnicas documentales) utilizando un método mixto mediante dos rondas presenciales (reunión de los participantes y aprobación de propuestas) y tres rondas enmascaradas (revisión del material de forma individual). El análisis se aplicó al ámbito de los diferentes niveles asistenciales.

Resultados: Se desarrolló una aplicación móvil basada en algoritmos consensuados que permite dar una orientación diagnóstica sobre el estado nutricional y dar soporte en la toma de decisiones mediante algoritmos basados en los criterios médicos ampliamente aceptados en el ámbito de la nutrición.

Conclusiones: la Herramienta de Evaluación Nutricional HEN se presenta como un apoyo a la práctica clínica habitual, facilitando el abordaje del estado nutricional de los pacientes en cualquier medio y entorno sanitario.

Palabras clave: Valoración nutricional; Aplicaciones móviles; Desnutrición.

\section{ABSTRACT}

Introduction: the use of so-called mobile applications is revolutionizing the way of working in clinical practice. Although, it is currently possible to identify a large number of these in the literature, there are no options similar to the presented proposal.

Objective: to develop a tool of simple use that allows a diagnostic orientation on the nutritional status, with normative support, available in different mobile platforms and oriented to facilitate the clinical praxis for the medical staff.

Methods: A consensus of experts (nominal group and documental techniques) was developed using a mixed method through face-toface sessions (meeting of the participants and approval of the proposals) and three masked rounds (review of the material in an individualized manner). The analysis was applied to the scope of the different levels of care.

Results: A mobile application was deployed taking into account consensual algorithms that enable a diagnostic orientation in the nutritional status and support in decision making.

Conclusions: the Nutritional Assessment Tool (HEN, in Spanish) is presented as a support to the regular clinical practice, facilitating the assessment of the nutritional status of patients in any environment and health environment.

Keywords: Nutrition Assessment; Mobile Applications; Malnutrition. 


\section{INTRODUCCIÓN}

Las aplicaciones móviles (Apps) son una tecnología que ya ha revolucionado el escenario de la práctica sanitaria, software diseñado para funcionar en teléfonos inteligentes y otros dispositivos móviles, su presencia en el mundo sanitario se ha abierto paso y progresivamente va aumentando su número y su utilidad. Se ha publicado que existen más de 97.000 aplicaciones médicas que se pueden descargar desde las plataformas móviles, de las que aproximadamente el 30\% están dirigidas a pacientes y profesionales, y $70 \%$ al público en general. A los profesionales, el uso de las Apps les permite agilizar su trabajo, tomar decisiones más rápidamente y con una menor tasa de error (1). Es de este modo como se ha dado inicio, hace ya años, a la llamada mHealth, es decir, a la provisión de servicios de la salud mediante el uso de dispositivos móviles.

En este ámbito, un claro ejemplo sería el soporte para tratar con la desnutrición en el entorno médico, una alteración que afecta entre el 10 y el 91\% (2,3) de los pacientes según los estudios, y que a pesar de los numerosos consensos de expertos (4-8), de diferentes asociaciones científicas, sigue estando sin resolver. Por ese motivo, se han desarrollado una batería de cuestionarios y herramientas para poder detectar y diagnosticar precozmente la desnutrición (8-12). Sin embargo sigue sin generalizarse su uso y sin incorporarse a la práctica clínica habitual de forma rutinaria y protocolizada. Probablemente, porque unos cuestionarios son muy largos, consumiendo mucho tiempo asistencial, en otros porque no se dispone de ellos en el momento propicio para poderlo realizar. En todo caso, el resultado es que a pesar de tener diferentes herramientas su uso no está generalizado. Sigue siendo necesario dar respuesta a esta necesidad de detectar y diagnosticar al paciente desnutrido precozmente. Y es en este punto donde surge este trabajo que pretende desarrollar una herramienta móvil basada en los consensos de expertos de las Sociedades científicas para servir como soporte en el cribado y en la orientación diagnóstica de los problemas relacionados con la desnutrición. La propuesta fue que esta herramienta tenía que contener y presentar además del criterio científico, las siguientes características: Accesibilidad (posibilidad de llevarla siempre y siempre disponible), fácil de administrar, que realizara cálculos y recomendaciones, y que emitiera una orientación diagnóstica y terapéutica, permitiendo continuidad y seguimiento evolutivo. Al mismo tiempo que fiabilidad, integralidad (que comprende todos los elementos o aspectos), integración, interacción clínica. Permitiendo un seguimiento y análisis de participación con la herramienta.

Por todo lo cual, el objetivo planteado fue desarrollar una herramienta de uso sencillo que permitiera una orientación diagnóstica sobre el estado nutricional, con soporte normativo, disponible en diferentes plataformas móviles y pensanda para la ayuda al profesional sanitario en la praxis clínica.

\section{MATERIAL Y MÉTODO}

Tomando como base los documentos publicados por las diferentes sociedades científicas: Documento SENPE-SEDOM (4), Consensus statement of the Academy of Nutrition and Dietetics/American Society for Parenteral and Enteral Nutrition $(5,6,7)$ ESPEN guidelines for nutrition screening 2002 (8), se realizó un consenso (grupo nominal y técnicas documentales) utilizando un método mixto mediante dos rondas presenciales (reunión de los participantes y aprobación de las propuestas) y tres rondas enmascaradas (revisión del material de forma individual) para establecer con una batería adecuada de ítems con relevancia clínica aceptada por los consensos consultados y a partir de ellos desarrollar los algoritmos de resultado o de decisión.

El grupo de trabajo estuvo conformado por un equipo multidisciplinar (farmacéuticos/as hospitalarios/as, médicos y enfermeras/os), quienes por su formación académica y experiencia profesional 
cumplían el criterio de elección establecido: ejercicio profesional, por más de 5 años, en puesto donde se traten pacientes desnutridos y/o con riesgo de desnutrición.

\section{Procedimiento}

El acuerdo se desarrolló en 7 fases que se detalla a continuación:

- Fase 1: Revisión bibliográfica de los antecedentes y consensos relacionados con la desnutrición relacionada con la enfermedad. Análisis de documentos relacionados con el hecho o contexto estudiado.

- Fase 2: Preparación de la documentación inicial y generación del primer algoritmo y su cuadro de gestión.

- Fase 3 (Enmascarada): Revisión del algoritmo y aporte de correcciones.

- Fase 4 (Presencial): Puesta en común de las aportaciones realizadas y corrección de los algoritmos.

- Fase 5 (Enmascarada): Nueva revisión de los documentos y nuevas aportaciones si se consideraba necesario.

- Fase 6 (Presencial): Aceptación de las últimas revisiones realizadas y generación del material corregido.

- Fase 7 (Enmascarada): Aprobación final del material por unanimidad: algoritmos.

\section{Ámbito de aplicación}

El análisis se aplicó al ámbito de la desnutrición relacionada con la enfermedad en cualquier ámbito asistencial.

A partir de los resultados obtenidos, todos los servicios fueron implementados de modo nativo para los entornos Android e iOS. De este modo, se desarrollaron aplicaciones diferentes en su codificación pero iguales en sus funcionalidades y conformes a lo presentado en el punto anterior. Para ello se recurrió a interfaces de acceso neutras desde un punto de vista tecnológico para el acceso a los datos de modo remoto.

\section{RESULTADOS}

Se establecieron cuatro dominios principales; cribado, valoración, requerimientos e indicación.

En el dominio de cribado del estado nutricional (ver figura 1) se consideraron: el sexo, la edad, el peso en $\mathrm{Kg}$, la altura en metros y el cálculo automatizado del IMC a partir de los anteriores parámetros los parámetros antropométricos, considerando para la edad, $<65$ años y $\geq 65$ años para la adecuada clasificación (ver figura 2). Se consideró necesario disponer de una aproximación a la talla y el peso de los pacientes cuando estas medidas no pudieran obtenerse directamente decidiendo aplicar las fórmulas que se consideraron más sencillas y más utilizadas en la práctica habitual (9). Además de dos preguntas, la primera sobre cómo está siendo actualmente su ingesta dietética referida a la última semana y la segunda a la evolución de su peso corporal (ver figura 3) sin que haya mediado ninguna acción voluntaria para disminuir su peso corporal (10). Esta dimensión se concluye con una decisión que indica según el algoritmo que no necesita valoración lo que significa que no se ha detectado riesgo de desnutrición y en el caso contrario informa de que necesita valoración, esto ocurre si alguno de los parámetros encuestados presenta riesgo de desnutrición. 
Figura 1. Pantalla del Cribado Nutricional

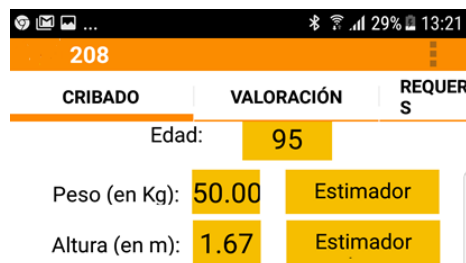

IMC: 17,93 . Su estado es Delgadez moderada.

¿Como es su ingesta actualmente?
La mitad de lo habitual
¿Ha perdido peso ultimamente?
Sí
I cuánto tiempo ha perdido peso? Un mes
¿Cuanto peso perdió (en kg.)? $\quad 5 \mid$

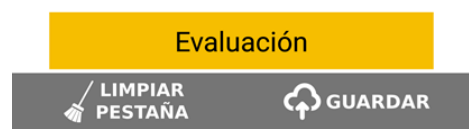

Figura 2. Clasificación del Índice de Masa Corporal

\begin{tabular}{|l|l|}
\hline $\begin{array}{l}\text { Clasificación del IMC } \\
<65 \text { años }\end{array}$ & \\
\hline Desnutrición severa & $<16,50$ \\
\hline Desnutrición leve & $16,50-16,99$ \\
\hline Delgadez aceptable & $17,00-18,49$ \\
\hline Normal & $\mathbf{1 8 , 5 0 - 2 4 , 9 9}$ \\
\hline Sobrepeso & $25,00-29,99$ \\
\hline Obesidad Grado I & $30,00-34,99$ \\
\hline Obesidad Grado II & $35,00-39,99$ \\
\hline Obesidad Grado III & $40,00-49,99$ \\
\hline Obesidad Grado IV & $\geq 50,00$ \\
\hline
\end{tabular}

\begin{tabular}{|l|l|}
\hline $\begin{array}{l}\text { Clasificación del IMC } \\
\text { para } \mathbf{6 5} \text { años }\end{array}$ & \\
\hline Desnutrición severa & $<16,50$ \\
\hline Desnutrición moderada & $16,50-16,99$ \\
\hline Desnutrición leve & $17,00-18,49$ \\
\hline Delgadez aceptable & $18,50-21,99$ \\
\hline Normal & $\mathbf{2 1 , 9 9 - 2 6 , 9 9}$ \\
\hline Sobrepeso & $27,00-29,99$ \\
\hline Obesidad Grado I & $30,00-34,99$ \\
\hline Obesidad Grado II & $35,00-39,99$ \\
\hline Obesidad Grado III & $40,00-49,99$ \\
\hline Obesidad Grado IV & $\geq 50,00$ \\
\hline
\end{tabular}


Figura 3. Valoración de la perdida de peso en función del tiempo

\begin{tabular}{|l|l|l|l|l|l|}
\hline $\begin{array}{l}\text { \% Pérdida de } \\
\text { Peso/Tiempo }\end{array}$ & Periodo & Valor normal & $\begin{array}{l}\text { Desnutrición } \\
\text { leve }\end{array}$ & $\begin{array}{l}\text { Desnutrición } \\
\text { moderada }\end{array}$ & $\begin{array}{l}\text { Desnutrición } \\
\text { severa }\end{array}$ \\
\cline { 2 - 6 } & 1 semana & $<1 \%$ & $1-2 \%$ & $2 \%$ & $>2 \%$ \\
\cline { 2 - 6 } & 1 mes & $<2 \%$ & $<5 \%$ & $5 \%$ & $>5 \%$ \\
\cline { 2 - 6 } & 2 meses & $<3 \%$ & $5 \%$ & $5-10 \%$ & $>10 \%$ \\
\cline { 2 - 6 } & 3 meses & $<7,5 \%$ & $<10 \%$ & $10-15 \%$ & $>15 \%$ \\
\hline
\end{tabular}

En la valoración nutricional (figura 4) se tienen en cuenta, además de las variables que ya se han introducido, los parámetros analíticos (10-12) proteínas totales (g/dl), albúmina (g/dl), linfocitos (células/mm3) y colesterol total ( $\mathrm{mg} / \mathrm{dl}$ ), adoptando los puntos de corte del Consenso SEDOM-SENPE (4) y para las proteínas totales el de Villalobos et al. (12). Estableciendo diferentes combinaciones de los parámetros analíticos y de las variables antropométricas y dietéticas recogidas en la fase de cribado se concluyen los diferentes estados de desnutrición así como es tipo de la misma apoyándose en la predominancia de unos u otros parámetros (4).

Figura 4. Dominio de Valoración Nutricional

\begin{tabular}{|c|c|c|}
\hline \multirow{3}{*}{ 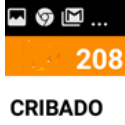 } & \multicolumn{2}{|c|}{ * } \\
\hline & & \\
\hline & VALORACIÓN & $\begin{array}{l}\text { REQUERIMI } \\
\mathrm{S}\end{array}$ \\
\hline
\end{tabular}

Proteinas totales (en g/ 6.50

Albumina (en g/dl): 2.74

Linfocitos (en u/mm3): $\quad 510.0($

Colesterol (en mg/dl): $\quad 168$

\section{Evaluación}


En el dominio de requerimientos, calcula mediante la fórmula predictiva de las ecuaciones de Harris-Benedict revisada por Mifflin y St Jeor (13), en 1990, considerando el peso real. Se valora la presencia de enfermedad, aplicando los criterios de Long adaptados para una mejor comprensión (14) y para ajustar por la actividad física se ha utilizado el factor de actividad física (FAF) (15).

Para el dominio de indicación se han considerado la posibilidad de alimentación oral, las dificultades para masticar, la presencia de disfagia, el tipo de disfagia y el grado de disfagia, además del porcentaje aproximado de requerimientos que cubre al día, poniendo como punto de corte el $75 \%$ de los requerimientos para recomendar una intervención nutricional que abarca desde dieta de texturas modificadas hasta nutrición parenteral (figura 5)

Figura 5. Algoritmo de decisión sobre la Indicación de Intervención Nutricional

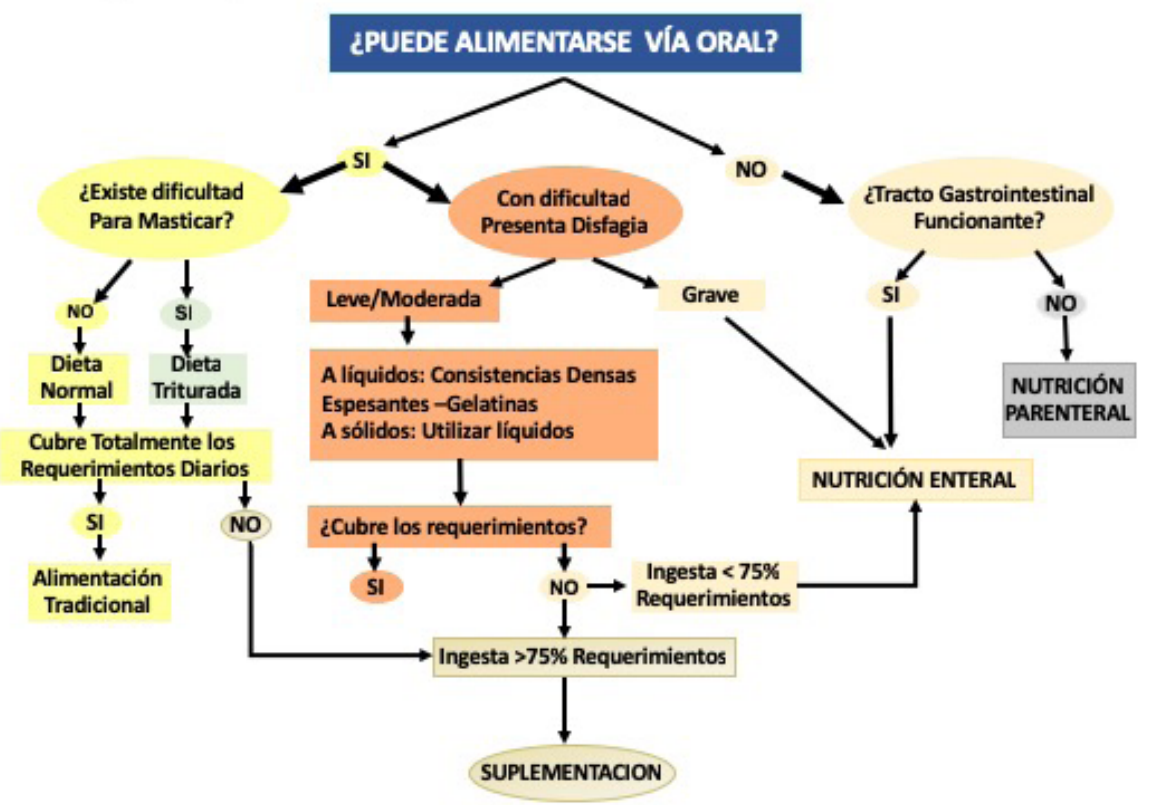

\section{DISCUSION}

La práctica clínica habitual requiere el manejo fluido de consensos y criterios médicos ampliamente respaldados en el dominio. Esto requiere un esfuerzo de puesta al día y la realización de cálculos matemáticos, que si bien no son necesariamente muy complejos, pueden entorpecer, enlenteciendo el normal desarrollo de la práctica clínica habitual de los profesionales sanitarios. Y, es en este contexto, en la práctica de la actividad clínica con el propio paciente, a pie de cama, donde aplicaciones como la presentada pueden ser de gran valor para profesional.

Esta herramienta, tal y como ha sido diseñada, supone una ayuda de campo para el profesional a la hora de estimar ciertos parámetros y condiciones del paciente. El profesional no necesitará 
manejar de primera mano los consensos médicos para cribar o clasificar pacientes sino que los tendrá disponibles en los algoritmos de esta herramienta. Además, dadas las posibilidades de la herramienta para gestionar datos de múltiples pacientes, ofrece una oportunidad para hacer seguimiento de los mismos y poder tener en cuenta su evolución clínica ya que permite descargar en una hoja CSV -valores separados por comas- (fácilmente convertible en Excel) los datos de todos los pacientes valorados por el profesional.

Los autores, además, consideran que este tipo de herramienta abre la puerta para nuevos tipos de soluciones en el ámbito del eHealth que pueden liberar el potencial de las nuevas técnicas de STEM (Science, Technology, Engineering and Mathematics). En particular, como línea futura, los autores se plantean la posibilidad de implementar algoritmos de Machine Learning (Aprendizaje automático) para permitir la clasificación inteligente de pacientes en función de parámetros ocultos.

\section{CONCLUSIONES}

La Herramienta de Evaluación Nutricional (HEN) se presenta como un apoyo a la práctica clínica habitual, facilitando el abordaje del estado nutricional de los pacientes en cualquier medio y entorno sanitario.

\section{BIBLIOGRAFIA}

1. Velsen L, Beaujean DJ, Gemert-Pijnen JE. Why mobile health app overload drives us crazy, and how to restore the sanity. BMC Med Inform Decis Mak 2013;13:23. PMID: 23399513; DOI: 10.1186/1472-6947-13-23

2. Pérez de la Cruz A, Lobo Támer G, Orduña Espinosa R, Mellado Pastor C, Aguayo de Hoyos E, Ruiz López MD. Desnutrición en pacientes hospitalizados: prevalencia e impacto económico. Med Clin (Barc). 2004;123(6):201-6. PMID: 15282072

3. Wanden-Berghe C, Cases Alarcón C, Santacruz Carmona N, García A, Granell L, Gonzalez Valls T, et al. Prevalencia de Desnutrición en los Pacientes de Hospitalización a Domicilio. Hosp Domic 2018;2(4):137-42. DOI: 10.22585/hospdomic.v2i4.54

4. Alvarez J, Del Río J, Planas M, García Peris P, García de Lorenzo A, Calvo V, et al; Grupo de Documentación de SENPE. Documento SENPE-SEDOM sobre la codificación de la desnutrición hospitalaria. Nutr Hosp. 2008;23(6):536-40. PMID: 19132260

5. White JV, Guenter P, Jensen G, Malone A, Schofield M; Academy of Nutrition and Dietetics Malnutrition Work Group; ASPEN Malnutrition Task Force; ASPEN Board of Directors. Consensus statement of the Academy of Nutrition and Dietetics/American Society for Parenteral and Enteral Nutrition: characteristics recommended for the identification and documentation of adult malnutrition (undernutrition). J Acad Nutr Diet. 2012;112(5):730-8. PMID: 22709779; DOI: 10.1016/j. jand.2012.03.012

6. White JV, Guenter P, Jensen G, Malone A, Schofield M; Academy Malnutrition Work Group; ASPEN Malnutrition Task Force; ASPEN Board of Directors. Consensus statement: Academy of Nutrition and Dietetics and American Society for Parenteral and Enteral Nutrition: characteristics recommended for the identification and documentation of adult malnutrition (undernutrition). JPEN J Parenter Enteral Nutr. 2012;36(3):275-83. PMID: 22535923; DOI: 10.1177/0148607112440285 
7. Mueller C, Compher C, Ellen DM; American Society for Parenteral and Enteral Nutrition (ASPEN) Board of Directors. ASPEN clinical guidelines: Nutrition screening, assessment, and intervention in adults. JPEN J Parenter Enteral Nutr. 2011;35(1):16-24. PMID: 21224430; DOI: $10.1177 / 0148607110389335$

8. Kondrup J1, Allison SP, Elia M, Vellas B, Plauth M; Educational and Clinical Practice Committee, European Society of Parenteral and Enteral Nutrition (ESPEN). ESPEN guidelines for nutrition screening 2002. Clin Nutr. 2003;22(4):415-21. PMID: 12880610

9. Elia M (chairman and editor); British Association for Parenteral and Enteral Nutrition (BAPEN): Advisory Group on Malnutrition. Guidelines for detection and management of malnutrition. Maidenhead, UK: BAPEN; 2000.

10. García de Lorenzo A, Álvarez J, Calvo M, Ulíbarri Jl, Del Río C, Galbán P, et al. Conclusiones del II foro de debate SENPE sobre: Desnutrición Hospitalaria. Nutr Hosp. 2005;20(2):82-7. PMID: 15813390

11. Ulibarri JI, González-Madroño A, de Villar MAL, González P, González B, Mancha A, et al. CONUT: A tool for controlling nutritional status - First validation in a hospital population. Nutr Hosp. 2005;20(1):38-45. PMID: 15762418

12. Villalobos Gámez JL, García-Almeida JM, Guzmán de Damas JM, Rioja Vázquez R, Osorio Fernández D, Rodríguez-García LM, et al. Proceso de INFORNUT: validación de la fase de filtro -FILNUT- y comparación con otros métodos para la detección de la desnutrición hospitalaria temprana. Nutr Hosp. 2006;21(4):491-504. PMID: 16913209

13. Mifflin MD, St Jeor ST, Hill LA, Scott BJ, Daugherty SA, Koh YO. A new predictive equation for resting energy expenditure in healthy individuals. Am J Clin Nutr. 1990;51(2):241-7. PMID: 2305711; DOI: 10.1093/ajcn/51.2.241

14. Long CL, Schaffel N, Geiger JW, Schiller WR, Blakemore WS. Metabolic response to injury and illness: estimation of energy and protein needs from indirect calorimetry and nitrogen balance. JPEN J Parenter Enteral Nutr. 1979;3(6):452-6. PMID: 575168; DOI: 10.1177/014860717900300609

15. Vazquez C, De Cos Blanco I, Lopez- Mondedeu C. Alimentación y nutrición: Manual-teórico práctico. $2^{a}$ ed. Madrid, España: Diaz de Santos; 2005. 